\title{
3D Trilateration Localization using RSSI in Indoor Environment
}

\author{
Nur Diana Rohmat Rose ${ }^{1}$, Low Tan Jung ${ }^{2}$ \\ Department of Computer and Information Sciences \\ Universiti Teknologi PETRONAS, Perak, Malaysia
}

\author{
Muneer Ahmad ${ }^{3}$ \\ Faculty of Computer Science and Information Technology \\ Universiti Malaya, Kuala Lumpur, Malaysia
}

\begin{abstract}
Received Signal Strength Indicator (RSSI) is one of the most popular technique for outdoor and indoor localization. There are many previous researches on RSSI-based indoor localization systems. However, most of them lack a solid classification method that reduce localization errors with better accuracy. This paper will focus on indoor localization methods to provide a technological perspective of indoor positioning systems. This paper proposes an indoor localization by using 3D trilateration method to locate target tags from RFID readers that used RSSI measurements for range determination. There will be six test cases for each reader. This system can track any target within the selected area with less localization error.
\end{abstract}

Keywords-RSSI; RFID; Indoor Positioning System (IPS); trilateration; $3 D$ localization

\section{INTRODUCTION}

Localization systems are a significant permissive technology nowadays and becoming one of the crucial part of our daily life. It can be divided into outdoor and indoor environment. Global Navigation Satellite System (GNSS) or Global Positioning System (GPS) is central to the various types of localization technologies used by a wide range of people and groups in our society. It is a satellite navigation systems that provide autonomous geospatial positioning with global coverage. GNSS is almost similar to GPS, but there are a few key differences between the two. However, the precise functions of GPS has its limits which it does not work well in indoor environment due to the attenuated and scattered signals from the satellites by roofs, floors, walls, and other objects [1]. In order to overcome this issues, Indoor Positioning System (IPS), a revolutionary technology is used to locate people or objects within indoor environment such as at underground locations, parking garages, and multistory buildings.

There are a wide range of IPS technologies used to track and locate people or objects in indoor environment such as Wireless Local Area Network (WLAN), Radio-Frequency Identification (RFID), ultrasonic, Bluetooth, and UltraWideband (UWB) [2]. However, IPS technologies cannot utilize GNSS for location detection unlike outdoor positioning systems that can achieve 1-3 meter accuracies through satellites [3].

The ability to locate people accurately and quickly in onfire buildings is quite critical to establish effective fire emergency response operations [4]. In a recent study [5], a series of interviews with the first responders assessed the value of the indoor location information. It was noticed that information on indoor location was among the most needed items of information when reacting to building fire incidents. Fire statistics show that 369,500 fires or $76 \%$ of all structure fires occurred in home structures (family homes and apartments), a slight increase of $1.2 \%$ were registered in the US in 2013, resulting in 2,755 civilian fire deaths [6]. Recently, more attention has been given to indoor positioning systems for multistory buildings. Such systems need not only to describe the coordinates $(\mathrm{x}, \mathrm{y})$ but also the floor where the object is located.

\section{INDOOR LOCALIZATION}

\section{A. Methods}

Most technologies nowadays are focusing on accurate real time object tracking and localization within buildings [7]. Thus, the rapid growth of indoor localization technologies has become an important demand for some markets. Researches on IPS are still widely conducted in order to improve the performance of localization technologies. A large variety of methods, techniques, principles, and devices are used to provide indoor positioning data in the form of signal strength or range. A basic indoor localization should have a reference sensor node with a known position and a target to be located [8].

Lateration/Trilateration/Multilateration. All these terms refer to a position determined by distance measurement. Lateration is the most common method to define an object's position by measuring its distance from multiple reference points. It uses the known distance of at least three fixed points in 2D space, or four fixed points in 3D space to determine an object's position [8]. Trilateration works by finding a series of circles that intersect with each other. Techniques based on the measurement of the signal propagation such as Time of Arrival (ToA), Time of Flight (ToF), Time Difference of Arrival (TDoA), and RSSI are defined as lateration techniques [9].

Time of Arrival (ToA) or Time of Flight (ToF) is the amount of time a signal takes to travel from a transmitter to a receiver. Because the rate of signal propagation is constant and known, a signal's travel time can be used to measure distance directly [10]. However, the accuracy of the TOAbased methods also suffers from significant multipath conditions in indoor environment caused by diffraction of the radio frequency (RF) signal from objects such as walls and floors. The distance from the reference point can be calculated using this simple equation: 
$d=c *\left(t_{\text {arrive }}-t_{\text {sent }}\right)$

where $c$ is the speed of light. In $2 \mathrm{D}$, this leads to the following equation:

$d=\sqrt{\left(x_{\text {ref }}-x\right)^{2}+\left(y_{\text {ref }}-y\right)^{2}}$

where $\left(x_{r e f}, y_{\text {ref }}\right)$ is the known position of the reference point. Once this set is calculated for enough reference points (at least three points for 2D or at least four points for 3D), the exact position of the target can be calculated by finding the intersection.

Time Difference of Arrival (TDoA) does not require the time that the signal was sent from the target, only the time the signal was received and the speed that the signal travels [11]. Once the signal is received at two reference points, the difference in arrival time can be used to calculate the difference in distances between the target and the two reference points. This difference can be calculated using the equation:

$\Delta d=c *(\Delta t)$

where $c$ represent the speed of light and $\Delta t$ is the difference in arrival times at each reference point. In $2 \mathrm{D}$, this leads to the following equation:

$\Delta d=\sqrt{\left(x_{2}-x\right)^{2}-\left(y_{2}-y\right)^{2}}-\sqrt{\left(x_{1}-x\right)^{2}-\left(y_{1}-y\right)^{2}}$

where $\left(x_{1}, y_{1}\right)$ and $\left(x_{2}, y_{2}\right)$ are the known positions of the beacons.

Received Signal Strength Indicator (RSSI) measures the amount of power present in a radio signal that a RF client device receives from an access point or router. One way to determine the efficiency of a communication link is by measuring the signal strength at the receiving antenna. If a transmitter is moved closer to a receiver, the strength of the transmitted signal increases at the receiving antenna. Otherwise, the signal strength at the receiving antenna decreases if a transmitter is moved further away. RSSI is measured in $\mathrm{dBm}$, with a higher negative value (in $\mathrm{dBm}$ ) indicating a weaker signal [12].

Angulation/Triangulation involves measuring angles and is used to measure unknown distances. This can be done by establishing a baseline length. From each point in Fig. 1, angles of distant points can be measured. Triangulation determine the distances by forming triangles from the three points, based on the lengths and angles measured. The Angle of Arrival (AoA) technique can be classified under this method.

Angle of Arrival (AoA) estimates the target's location from the intersection of the several pairs of angle direction lines, each formed by the circular radius from a base station. This method requires only two measuring units for $2 \mathrm{D}$ and three measuring units for 3D. AoA does not require synchronization between the measuring units. Furthermore, it is based on direction, which received signal arrives from one device to another [13]. However, this method does not work efficiently in indoor environment since the accuracy and precision decreases when there are signal reflections from surrounding objects.

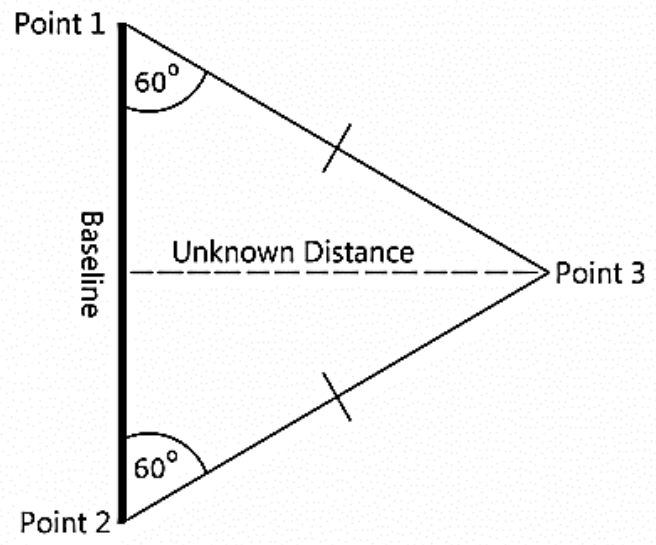

Fig. 1. Triangulation Localization Method.

Fingerprinting. Indoor environment produces many significant noises such as multipath fading, signal occlusions due to walls or objects, and signal diffractions depending on the object's material. Fingerprinting is the most popular localization method since it has higher accuracy compared to other methods. Most indoor localization approaches have adopted a matching fingerprint as the basic location determination scheme. The main idea is to collect scene features (fingerprint) from the surrounding signatures at each location in the areas of interest and then build a fingerprint database [7].

Proximity is the simplest method for localization. This localization method belongs to the range-free localization group. The assumption based on this method is, if the point is within the range of a known station, we can calculate the point's location to the known station. The location of user can be estimated to the position of access point for connection based on wireless communication. This method was utilized by the Cell-ID method standardized in GSM cellular systems, followed by RFID and Bluetooth-based systems [14].

Dead Reckoning (DR) is one of the useful method to overcome limitations where GPS or GNSS signals does not propagate very well within some environment such as underground passages, basements, or tunnels. When a vehicle pass through some environment where very strong multipath propagation occurs, GPS or GNSS signals cannot be received. By utilizing dead reckoning capabilities, the receiver will consistently output position with some hybrid positioning with GPS or GNSS signals. Information from various sensors are used to determine the current position which enables high accuracy localization. This method is widely used in automotive navigation systems.

\section{B. Technologies}

In this section, we present some representative examples and the description of indoor localization technologies which are Infrared Radiation (IR), Radio-Frequency Identification (RFID), Bluetooth, Wireless Local Area Network (WLAN), and Ultra-Wideband (UWB).

Infrared Radiation (IR) is an electromagnetic radiation (EMR) that is invisible to the human eye, although longer infrared waves can be sensed as heat. Infrared light, with 
wavelengths longer that the visible light can be used in wired or wireless operations depending on the situation [15]. Lower frequency light such as infrared light is usually used in fiber optic cables to transmit data due to the ability to travel farther down fiber optic lines with less power (intensity) required than higher frequency light. The main advantages of IR is it requires minimum power to operate and can be set up at a low cost [16]. Infrared transmission is also a secure way to transfer data between devices as the signal cannot pass beyond a room or chamber. However, the disadvantages of infrared are it can be used for a small range distance and the signals can be interpreted by objects, people, and impacted by weather conditions.

Radio-Frequency Identification (RFID) is a technology with the use of electromagnetic fields or radio waves to wirelessly identify and capture information of a tag attached to the object. There is a wide range of RFID applications in various industries such as automobile, manufacturing, retail, education, and healthcare. The advantages of RFID tags are it can be read without the line of sight, multiple tags can be read simultaneously, and the ability to read data without visual access. In spite of that, privacy is a main concern with the use of RFID on products as it can be easily tapped or intercepted and the external electromagnetic interference can limit the RFID remote reading [13].

Bluetooth is a short-range wireless communication technology that allows two different Bluetooth-enabled devices to connect by using low-energy radio waves in order to send the data [17]. Bluetooth can be considered as a wireless replacement of cables as common as Wi-Fi but with less power consumption and less implementation cost than Wi-Fi [18]. Bluetooth is used for voice and data transfer with a better range to be compared with Infrared since it is able to avoid interference from other wireless devices. However, there are some limitations of Bluetooth which it can lose connection in certain conditions and allow only short range communication between devices.

Wireless Local Area Network (WLAN) positioning refers to the process of allowing devices to connect and communicate through a WLAN infrastructure. A WLAN can provide a connection to the wider Internet through a gateway in order to achieve high flexibility for ad hoc communication. WLANs are based on IEEE 802.11 standards which refers to a family of specifications developed by the IEEE for WLAN technology [19]. WLAN positioning system use RSSI values from the Access Points (AP). Mobile devices will measure the signal strength receives from the APs. Nevertheless, APs are quite expensive to be compared with wires and hubs. In terms of flexibility, the nodes can communicate without further restriction. It is also a good alternative in terms of accuracy, precision, and cost since we can leverage on the existing infrastructure.

Ultra-Wideband (UWB) is an ultra-low power wireless technology for transmitting large amounts of digital data over a short distances. The UWB data rate is significantly higher than the Bluetooth and Wi-Fi technologies. The main advantage of UWB is the transmission using UWB are very secure due to the low power density that limits the interference potential with conventional radio systems. Furthermore, it is a promising indoor positioning technology which provides high accuracy since its bandwidth is very high that it can allow very high data throughput for communications devices [20]. It is also able to penetrate different type of materials easily. When there are obstacles, we can call this non-line-of-sight (NLOS). However, this technology requires higher initial implementation cost with slower adoption rate [21].

\section{Methodology}

\section{A. Proposed Procedures}

In this research, MATLAB software is used to evaluate the performance of the proposed localization method virtually based on the mathematical models in order to track and locate target tags within the selected area from RFID readers. The simulation can be categorized into two main phases which are 3D localization and multistory localization. Fig. 2 shows the phases in this research. However, this paper will discuss the simulation results of phase 1 only which is $3 \mathrm{D}$ localization.

Fig. 3 below shows RFID localization system block diagram. Signal strength of reference tags will be read from the RFID readers. In this research, the authors use the signal strength and reference position of readers from reference paper [22]. The RSSI values from RFID readers will be used for distance estimation between nodes by applying logdistance path loss model. To locate a target tag in $3 \mathrm{D}$ environment, trilateration localization algorithm will be applied. The target tag coordinates in $\mathrm{x}, \mathrm{y}$, and $\mathrm{z}$ plane will be located once we applied the localization algorithm. The localization accuracy can be determined by the analysis based on the number of positions that have been entered into the database.

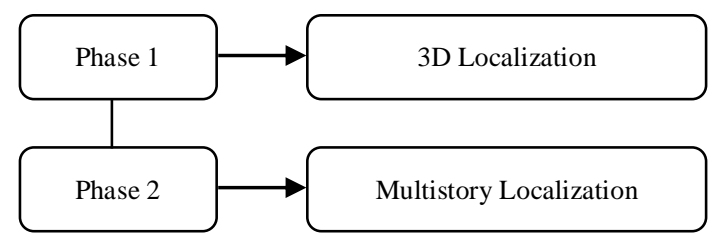

Fig. 2. Simulation Phases.

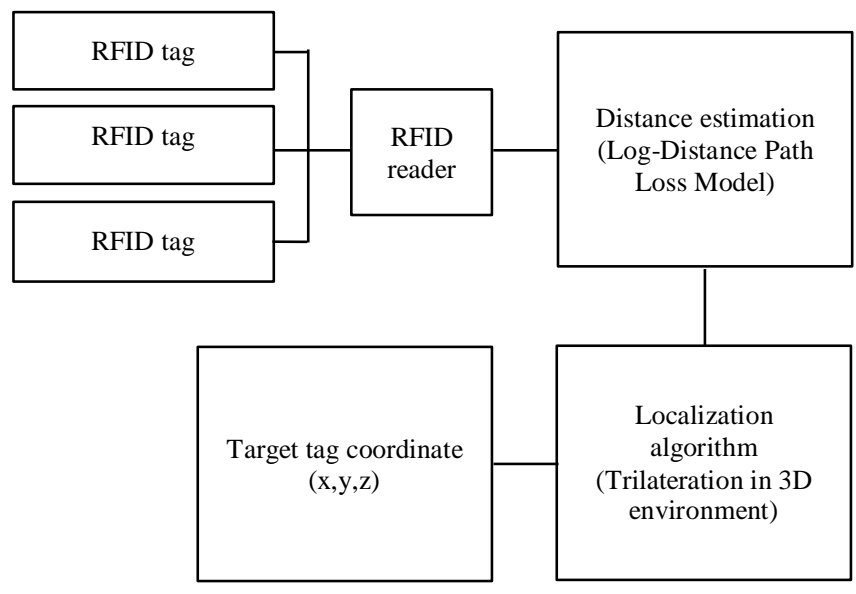

Fig. 3. RFID Localization System Block Diagram. 
The aim of this localization system is to estimate the position coordinate of a target node with the help of at least three reference nodes by using RSSI-based trilateration algorithm with an acceptable localization error. RSSI measures the signal power at the receiver. Based on the transmitted power, the propagation loss is calculated and the loss can be translated into distance estimation. Trilateration method helps to figure out the center point of three nodes surrounded by circles for which the distance and position is already known.

\section{B. System Flowchart}

The distances between readers and target tags can be calculated based on the RSSI values [23]. In this research, the authors use the signal strength and reference position of readers from reference paper [22] as shown in Table I. Factors of the RFID readers used in Table I are shown in the Table II below. Based on the RSSI values, we can estimate and calculate the distance between the nodes by using log-distance path loss model. Fig. 4 shows the system flowchart for this 3D trilateration localization.

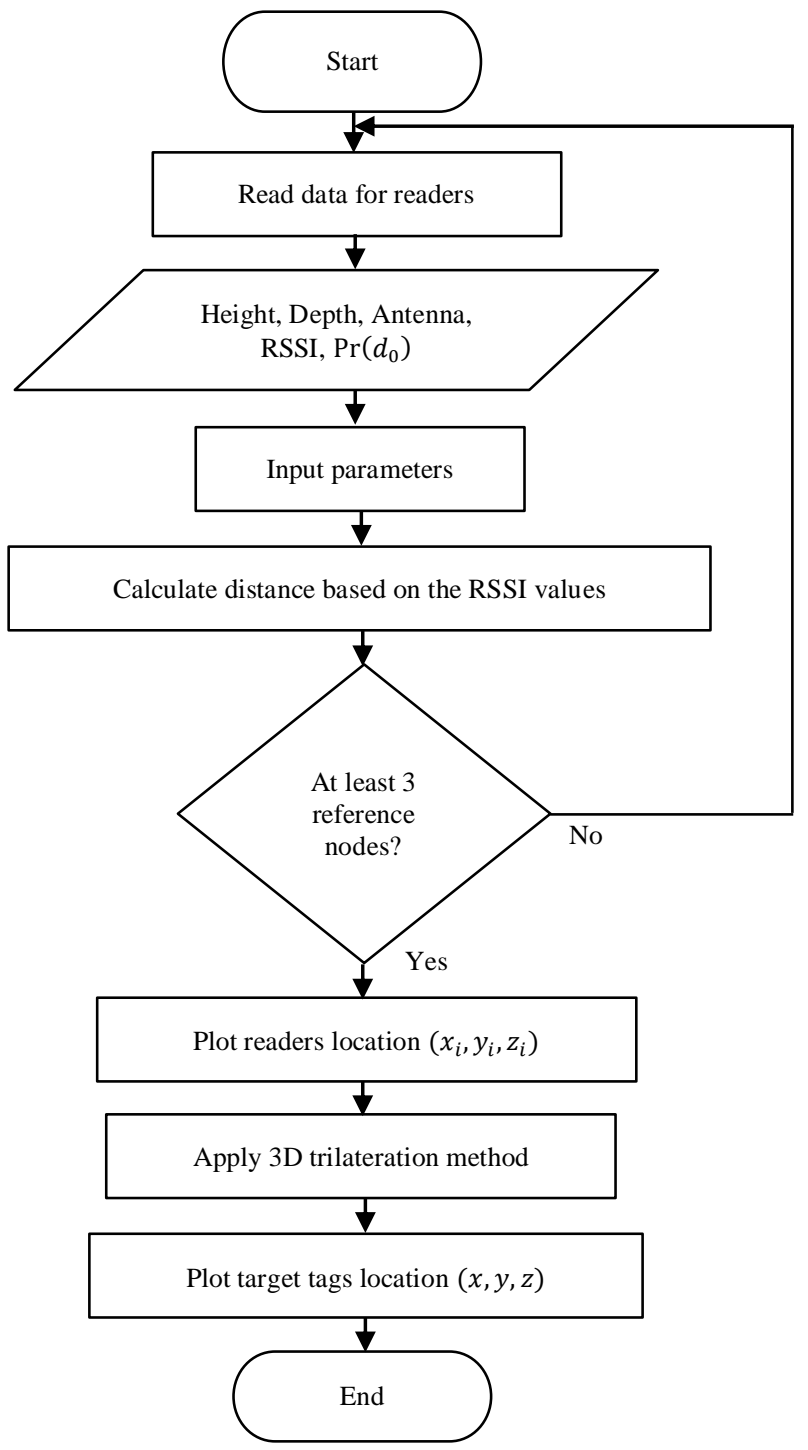

Fig. 4. System Flowchart.
TABLE. I. RFID READERS WITH CORRESPONDING DATA

\begin{tabular}{|c|c|c|c|c|c|}
\hline Reader & Height & Depth & Antenna & RSSI & $\operatorname{Pr}\left(d_{0}\right)$ \\
\hline \multirow{6}{*}{1} & 1 & 1 & 1 & -51 & 39.01 \\
\hline & 1 & 3 & 3 & -55 & 0 \\
\hline & 3 & 1 & 1 & -57 & 42.74 \\
\hline & 1 & 3 & 1 & -56 & 42.7 \\
\hline & 3 & 1 & 3 & -53 & 41.47 \\
\hline & 3 & 3 & 3 & -53 & 0 \\
\hline \multirow{6}{*}{2} & 1 & 3 & 1 & -60 & 41.47 \\
\hline & 1 & 3 & 3 & -53 & 0 \\
\hline & 3 & 1 & 1 & -63 & 42.74 \\
\hline & 3 & 1 & 3 & -56 & 42.7 \\
\hline & 1 & 1 & 1 & -53 & 44.16 \\
\hline & 3 & 3 & 3 & -52 & 0 \\
\hline \multirow{6}{*}{3} & 3 & 1 & 3 & -55 & 40.37 \\
\hline & 1 & 3 & 3 & -56 & 0 \\
\hline & 1 & 1 & 1 & -52 & 42.73 \\
\hline & 3 & 1 & 1 & -62 & 42.7 \\
\hline & 1 & 3 & 1 & -55 & 44.19 \\
\hline & 3 & 3 & 3 & -55 & 0 \\
\hline \multirow{6}{*}{4} & 1 & 3 & 3 & -57 & 40.37 \\
\hline & 3 & 3 & 3 & -58 & 0 \\
\hline & 1 & 3 & 1 & -61 & 38.46 \\
\hline & 1 & 1 & 1 & -54 & 43.9 \\
\hline & 3 & 1 & 1 & -60 & 44.19 \\
\hline & 3 & 1 & 3 & -53 & 0 \\
\hline
\end{tabular}

TABLE. II. FACTORS OF RFID READERS

\begin{tabular}{|c|c|c|c|}
\hline Factor & Symbol & Level & Description \\
\hline \multirow{3}{*}{ Height } & 1 & $0.4 \mathrm{~m}$ & Close to the ground \\
\hline & 2 & $1.2 \mathrm{~m}$ & $\begin{array}{l}\text { Far away from either the ground or the } \\
\text { ceiling }\end{array}$ \\
\hline & 3 & $2 \mathrm{~m}$ & Close to the ceiling \\
\hline \multirow{3}{*}{ Depth } & 1 & $0.2 \mathrm{~m}$ & Close to the wall \\
\hline & 2 & $0.6 \mathrm{~m}$ & $\begin{array}{l}\text { Neither too close nor too far away to } \\
\text { the wall }\end{array}$ \\
\hline & 3 & $1 \mathrm{~m}$ & Far away from the wall \\
\hline \multirow{3}{*}{ Antenna } & 1 & {$[\backslash /]$} & $\begin{array}{l}\text { Antenna A and B are both in the } \\
\text { vertical plane with } 45 \text { degrees to the } \\
\text { ground and } 90 \text { degrees in between }\end{array}$ \\
\hline & 2 & {$\left[L^{\prime}\right]$} & $\begin{array}{l}\text { Antenna A and B are both in the } \\
\text { vertical plane with A in the vertical } \\
\text { direction and B in the horizontal } \\
\text { direction }\end{array}$ \\
\hline & 3 & {$[\cdot \mid]$} & $\begin{array}{l}\text { Antenna A is in the horizontal plane } \\
\text { and pointing straight forward, while } \\
\text { antenna B is in the vertical plane and } \\
\text { in the vertical direction. }\end{array}$ \\
\hline
\end{tabular}


In order to calculate the distance between the nodes, the authors use the log-distance path loss model or known as penetration thru buildings model based on the RSSI values collected before [24]. Average received signal power decreases logarithmically with distance, whether in outdoor or indoor environment. Log-distance path loss model is a general propagation model. It can be used in both indoor and outdoor environment. The distance can be calculated using the equation:

$d=d_{0} * \exp \left(\frac{\operatorname{Pr}\left(d_{0}\right)-\operatorname{Pr}(d)}{10 n}\right)$

where $d_{0}$ is the near earth reference distance which we used its value as 1 meter for indoor environment, $\operatorname{Pr}\left(d_{0}\right)$ represent received power at reference distance $d_{0}$ and $n$ is the path loss exponent which its value is between 2 to 6 depending on the environment.

\section{3D Trilateration Method}

The distance calculated in part $B$ will be used to locate target tags location by trilateration. Trilateration localization algorithm will be applied in order to get the location coordinate output on the $\mathrm{x}, \mathrm{y}$, and $\mathrm{z}$ plane or in other words, in $3 \mathrm{D}$ localization. The quadratic equation for this trilateration localization algorithm is as follows:

$$
\begin{aligned}
& \left(x-x_{1}\right)^{2}+\left(y-y_{1}\right)^{2}+\left(z-z_{1}\right)^{2}=d_{1}{ }^{2} \\
& \left(x-x_{2}\right)^{2}+\left(y-y_{2}\right)^{2}+\left(z-z_{2}\right)^{2}=d_{2}{ }^{2} \\
& \left(x-x_{3}\right)^{2}+\left(y-y_{3}\right)^{2}+\left(z-z_{3}\right)^{2}=d_{3}{ }^{2} \\
& \left(x-x_{4}\right)^{2}+\left(y-y_{4}\right)^{2}+\left(z-z_{4}\right)^{2}=d_{4}{ }^{2}
\end{aligned}
$$

where $(x, y, z)$ is the estimated target tag coordinate, $\left(x_{i}, y_{i}, z_{i}\right)$ is the position of the readers and $d_{i}$ is the calculated distance from target tag to the four readers. Since the equations contain three unknowns and four equations, three equations are used to calculate unknowns. Fourth equation is taken as a reference to select the correct pair of variables.

\section{Simulation Setup}

In this research, simulation by using MATLAB was used for RFID readers to locate any target tag within the selected area based on the RSSI values. The simulation was carried out in a selected area with the dimension of $3 \mathrm{~m} \times 3 \mathrm{~m} \times 3 \mathrm{~m}$. There will be four RFID readers located in the area to locate or track any target tag in order to fulfil the objective of this simulation. Distance estimation from the four nodes to the target node is calculated by using the log-distance path loss model as shown in equation (5). In order to plot the location of the nodes in 3D localization, we will need to have at least three reference nodes. Trilateration localization algorithm as shown in equation (6), (7), (8), and (9) will be applied in order to get the location coordinate output of the estimated target tag in $3 \mathrm{D}$ localization.

\section{DiscUSSION}

Each red dot in the figures below symbolized the RFID readers. The position of the four RFID readers are located at $(1,1,1),(1,3,1),(3,1,3)$ and $(1,3,3)$. The location of the target tag shown by a green dot within the red dots (RFID readers).
There will be six test cases for each RFID reader. The position of the target tag for test case 1 is $(1.5289,1.5289,2.2739)$ as shown in Fig. 5. The green dot in Fig. 6 below showing position of the target tag.

For test case 2 and test case 6 , three reference nodes does not exist due to the path loss exponent. In the study of wireless communications, path loss can be represented by the path loss exponent, whose value is normally in the range of 2 to 4 . In some environments, such as buildings, stadiums and other indoor environments, the path loss exponent can reach values in the range of 4 to 6 . On the other hand, a tunnel may act as a waveguide, resulting in a path loss exponent less than 2. Path loss is usually expressed in $\mathrm{dB}$. So, we can conclude that the value of path loss exponent is between 2 to 6 depending on the environment.

For test case 3, there are two target tags located at $(1.5289,1.5289,2.2739)$ and $(1.99229,3.1260,2.0074)$ as shown in Fig. 7.

Fig. 8 shows the three target tags located at $(1.5289,1.5289,2.2739), \quad(1.99229,3.1260,2.0074) \quad$ and $(3.3015,3.3122,2.0000)$ for test case 4 .

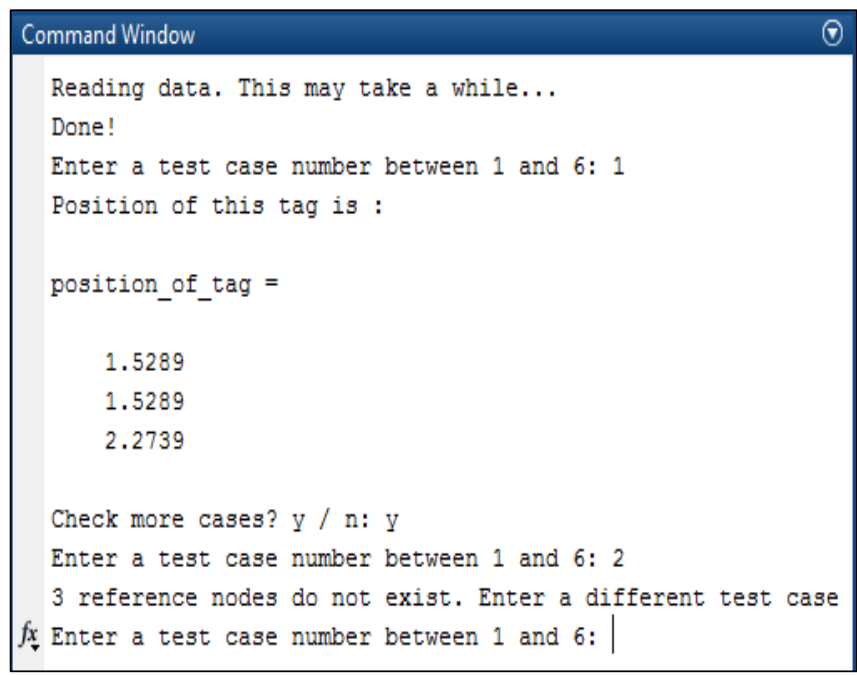

Fig. 5. Command Window for Test Case 1 and 2.

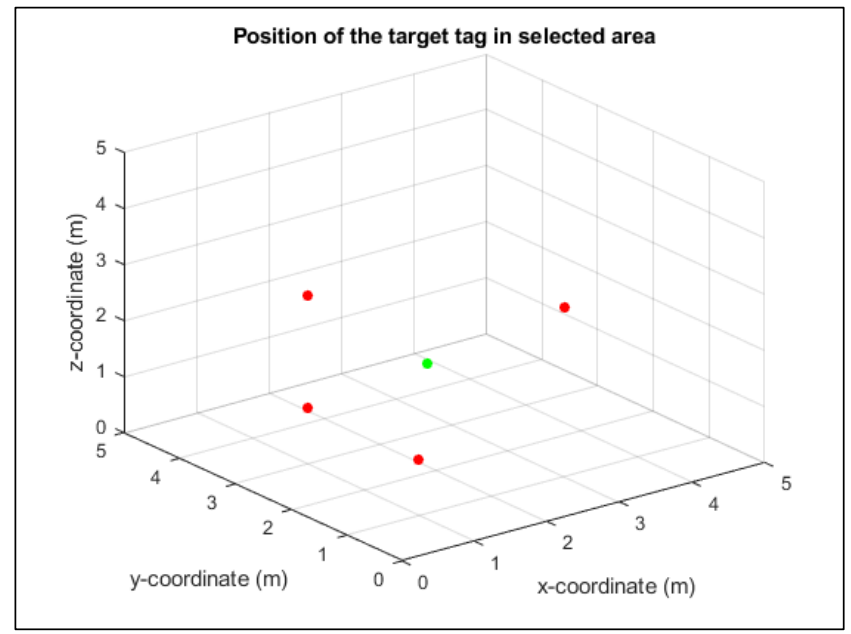

Fig. 6. 3D Trilateration with One Target Tag. 


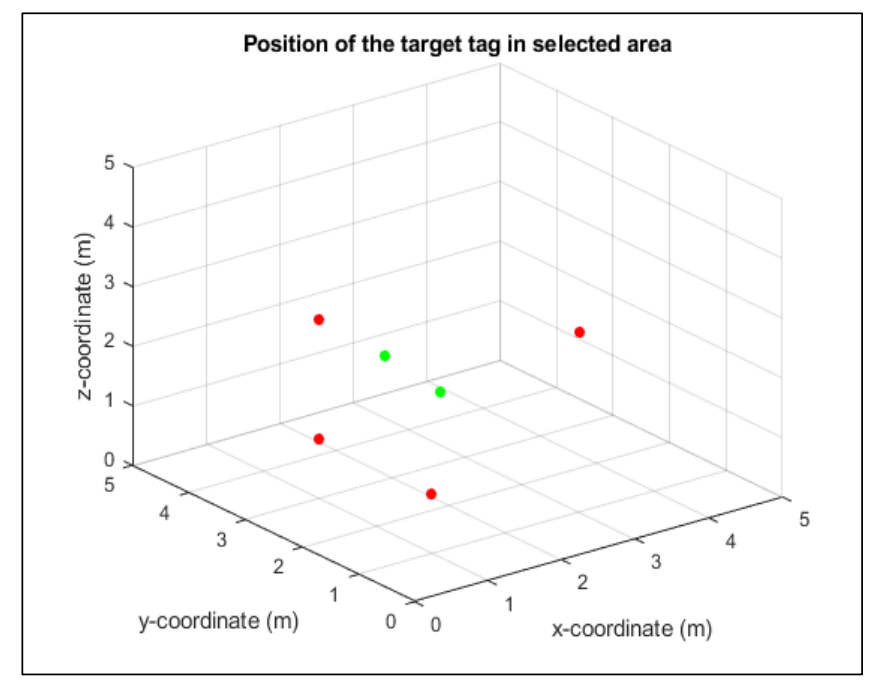

Fig. 7. 3D Trilateration with Two Target Tags.

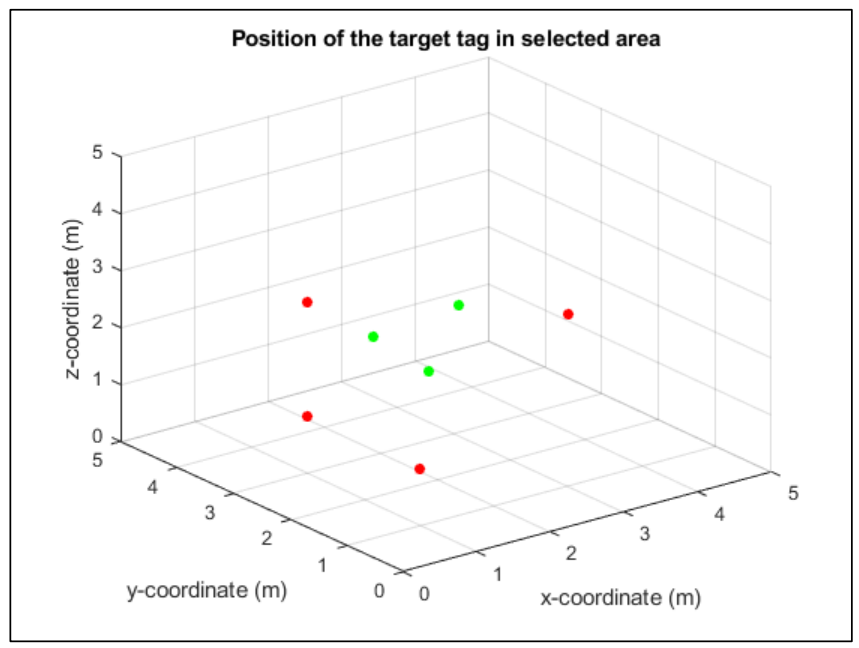

Fig. 8. 3D Trilateration with Three Target Tags.

Fig. 9 shows the location of four target tags for test case 5 at $\quad(1.5289,1.5289,2.2739), \quad(1.99229,3.1260,2.0074)$, $(3.3015,3.3122,2.0000)$ and $(1.9383,1.9383,4.4331)$.

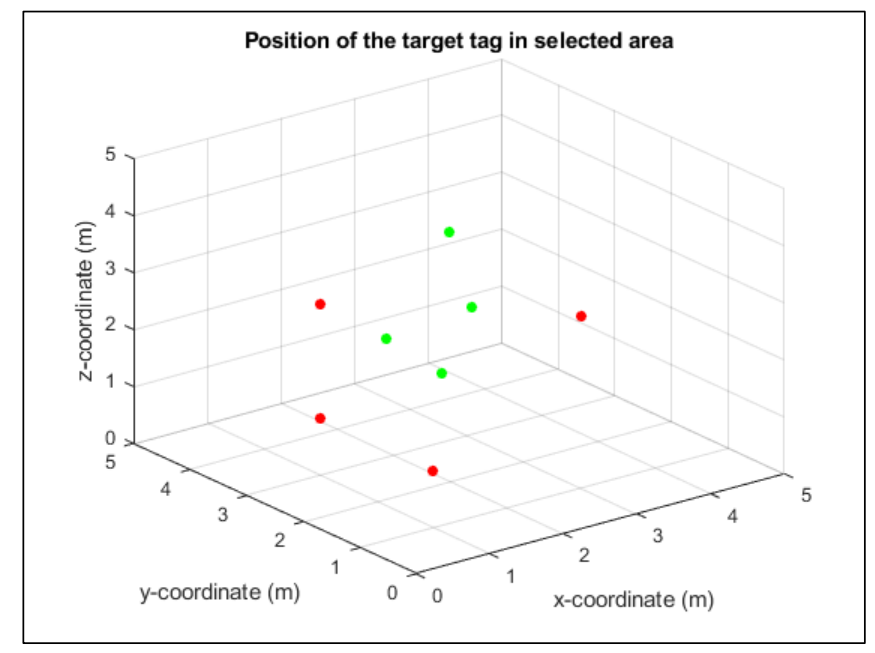

Fig. 9. 3D Trilateration with Four Target Tags.
This 3D trilateration localization simulation will be extended into the next phase which is multistory localization. The comparison between these two will be used in order to measure the accuracy of 3D indoor localization in multistory environment.

\section{V.CONCLUSION}

IPS use sensors and communication technologies to locate objects in indoor environment. IPS are attracting scientific and enterprise interest because there is a big market opportunity for applying these technologies. In this paper, trilateration method is used for 3D localization to locate target tag in the selected area accurately by using RSSI values. RSSI is a measure of the strength of a signal received from a tag. From the six test cases, we can conclude that RSSI signals is important to determine how close a target tag is to a reader antenna and to consider the real-time operation of sensor nodes. The RSSI values varies depending on the distance of the tag from the reader's antenna. There are several other factors to be considered when utilizing RSSI which are the orientation of the tag with respect to the reader's antenna, the material of the tag and blocking objects between the tag and the reader.

\section{FUTURE WORK}

Current surveillance systems for multistory building lack in supporting real-time monitoring of every point of a level in multistory buildings at all times. Solutions using wireless sensor networks, on the other hand, can gather sensory data values from all points of a building continuously, day and night to provide fresh and accurate data. However, sensor networks face serious obstacle which is lack of accurate indoor localization for multistory buildings. Recommendation for further studies in this area is to propose the best method for indoor localization as well as to prove that the method can determine accurate indoor localization for multistory building. While continuously preserving the goal of building monitoring, it is also to construct a system that shall regards the lack of accurate localization that may hinder the network performance.

\section{ACKNOWLEDGMENT}

The authors would like to thank the Department of Computer and Information Sciences, Universiti Teknologi PETRONAS for providing the information, guidance, facilities and financial support through Graduate Assistantship (GA) Scheme throughout this research activities.

\section{REFERENCES}

[1] M. Er Rida, F. Liu, Y. Jadi, A. A. A. Algawhari, and A. Askourih, "Indoor location position based on bluetooth signal strength," Proc. 2015 2nd Int. Conf. Inf. Sci. Control Eng. ICISCE 2015, pp. 769-773, 2015.

[2] T. Kim and E. J. Kim, "A novel 3D indoor localization scheme using virtual access point," Int. J. Distrib. Sens. Networks, vol. 2014, pp. $6-$ $11,2014$.

[3] L. Batistic and M. Tomic, "Overview of indoor positioning system technologies," 2018 41st Int. Conv. Inf. Commun. Technol. Electron. Microelectron. MIPRO 2018 - Proc., pp. 473-478, 2018.

[4] N. Li, B. Becerik-Gerber, B. Krishnamachari, and L. Soibelman, "A BIM centered indoor localization algorithm to support building fire 
emergency response operations," Autom. Constr., vol. 42, pp. 78-89, 2014.

[5] N. Li, Z. Yang, A. Ghahramani, B. Becerik-Gerber, and L. Soibelman, "Situational awareness for supporting building fire emergency response: Information needs, information sources, and implementation requirements," Fire Saf. J., vol. 63, pp. 17-28, 2014.

[6] M. J. Kenter, "Fire loss in the United States during 1989," Fire J. Boston, Mass., vol. 84, no. 5, 1990.

[7] Z. Farid, R. Nordin, and M. Ismail, "Recent advances in wireless indoor localization techniques and system," J. Comput. Networks Commun., vol. 2013, 2013.

[8] M. Md Din, N. Jamil, J. Maniam, and M. A. Mohamed, "Review of indoor localization techniques,” Int. J. Eng. Technol., vol. 7, no. 2.14, p. 201, 2018.

[9] D. Zhang, F. Xia, Z. Yang, L. Yao, and W. Zhao, "Localization technologies for indoor human tracking," 2010 5th Int. Conf. Futur. Inf. Technol. Futur. 2010 - Proc., no. 60903153, pp. 1-6, 2010.

[10] P. Shirke, A. Potgantwar, and V. M. Wadhai, "Analysis of RFID Based Positioning Technique Using Received Signal Strength and Directional Antenna Global Positioning System (GPS), Indoor Positioning System (IPS), Radio Frequency Identification (RFID), Received Signal Strength (RSS)," vol. 7, no. May, pp. 80-89, 2016.

[11] G. Kul, T. Özyer, and B. Tavli, "IEEE 802.11 WLAN based real time indoor positioning: Literature survey and experimental investigations," Procedia Comput. Sci., vol. 34, no. August, pp. 157-164, 2014.

[12] N. Rosli et al., “Jurnal Teknologi,” vol. 1, pp. 1-6, 2015.

[13] J. Kárník and J. Streit, "Summary of available indoor location techniques," IFAC-PapersOnLine, vol. 49, no. 25, pp. 311-317, 2016.

[14] C. Laoudias, A. Moreira, S. Kim, S. Lee, L. Wirola, and C. Fischione, "A survey of enabling technologies for network localization, tracking, and navigation," IEEE Commun. Surv. Tutorials, vol. 20, no. 4, pp. 3607-3644, 2018.

[15] R. F. Brena, J. P. García-Vázquez, C. E. Galván-Tejada, D. MuñozRodriguez, C. Vargas-Rosales, and J. Fangmeyer, "Evolution of Indoor Positioning Technologies: A Survey,” J. Sensors, vol. 2017, 2017.

[16] S. Ma, Q. Liu, and H. Tang, "An Overview of Location Semantics Technologies and Applications," Int. J. Semant. Comput., vol. 9, no. 3, pp. 373-393, 2015.

[17] I. Oksar, "A Bluetooth signal strength based indoor localization method," Int. Conf. Syst. Signals, Image Process., no. May, pp. 251254, 2014.

[18] Y. Zhuang, J. Yang, Y. Li, L. Qi, and N. El-Sheimy, "Smartphone-based indoor localization with bluetooth low energy beacons," Sensors (Switzerland), vol. 16, no. 5, pp. 1-20, 2016.

[19] S. Dhanalakshmi and M. Sathiya, "An Overview of IEEE 802.11 Wireless LAN Technologies," Int. J. Comput. Sci. Mob. Comput., vol. 4, no. 1, pp. 85-93, 2015.

[20] A. K. Paul and T. Sato, "Localization in wireless sensor networks: A survey on algorithms, measurement techniques, applications and challenges," J. Sens. Actuator Networks, vol. 6, no. 4, 2017.

[21] J. Wu, “DigitalCommons @ University of Nebraska - Lincoln ThreeDimensional Indoor RFID Localization System," 2012.

[22] J. Wu, "Three-Dimensional Indoor RFID Localization System," Univ. Nebraska -Lincoln, p. 199, 2012.

[23] O. G. Adewumi, K. Djouani, and A. M. Kurien, "RSSI based indoor and outdoor distance estimation for localization in WSN," Proc. IEEE Int. Conf. Ind. Technol., pp. 1534-1539, 2013.

[24] M. Region, "RSSI based Trilateral Indoor Localization System using Reference Tags of Radio Frequency Identification System," vol. 07, no. 02, pp. 276-279, 2018. 